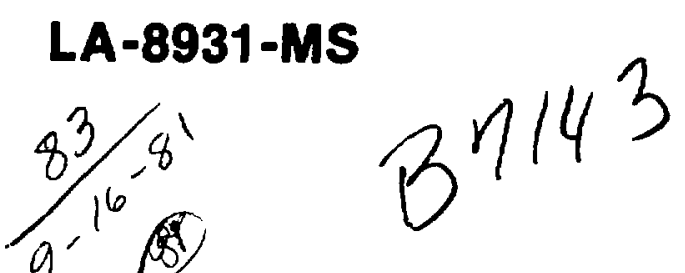

(2)

Wh. 3029

Radiative Transfer Approximations in

Stellar Pulsation Theory

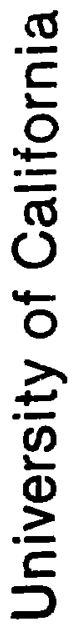


LA-8931-MS

UC-34b

Issued: August |9RI

\section{Radiative Transfer Approximations in Stellar Pulsation Theory}

Cecil G. Davis 


\section{CONTENTS}

ABSTRACT. .....................................

I. IntRODUCTION..............................

II. THE EQUATIONS OF NONLINEAR PULSATI IN THEORY............ 2

III. THE RADIATION TRANSFER EQUATION................. 6

IV. EXPANSION IN TERMS OF MOMENTS.................. 8

v. THE VARIABLE EDDINGTON FACTOR................... 11

VI. FREQUENCY DEPENDENCE. ........................ 13

VII. VELOCITY TERMS AND RETARDATION.................. 14

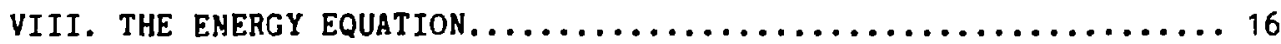

A. The "Partial Temperature" Approach............... 17

B. The "Multifrequency Gray" Approach................ 17

IX. THE "SPEC" CODE AND SOME APPLICATIONS............... 18

x. ConcLusions............................... 19

REFERENCES................................... 21

APPENDIX $\ldots \ldots \ldots \ldots \ldots \ldots \ldots \ldots \ldots \ldots \ldots \ldots \ldots \ldots \ldots \ldots \ldots \ldots \ldots \ldots$ 


\title{
RADIATIVE TRANSFER APPROXIMATIONS IN \\ STELLAR PULSATION THEORY
}

\author{
by
}

Cecil G. Davis

\begin{abstract}
Pulsation theory is discussed in light of recent improvements in the theory of radiative transfer and hydrodynamics with applications to Cepheids, RR Lyrae, and $W$ Virginis stars.
\end{abstract}

\section{INTRODUCTION}

From the work of Eddington, ${ }^{1}$ Zherakin, ${ }^{2}$ and $\operatorname{cox}$ and Whitney, ${ }^{3}$ we know the basic mechanisms causing the driving force for the pulsation observed in such variable stars as Cepheids, W Virginis, and RR Lyrae. In the ionization of hydrogen and helium, a "heat valve" dams up the energy flow on compression and then releases this energy to drive the pulsation on expansion. The theory of nonlinear pulsation was given a great boost with the development of large computers. The original work if Christy ${ }^{4}$ and Cox. Brownlee, and Eilers ${ }^{5}$ utilized a large computer (IBM 704) to solve the coupled set of nonitinear pulsation equations.

We review the development of an improved theory of nonlinear pulsation based on the use of the multifrequency variable Eddington approximation of radiative transfer theory. Around 1965 a number of people, including a student of Christy's, John Castor (Consuitant at Los Alamos), and Group j-15 (Los Alamos) began to include radiative transfer, replacing the previously applied diffusion theory, in calculations of nonlinear pulsation. In Sec. II, we describe the basic nonlinear pulsation equations and the driving mechanisms. In Secs. III 
through $v$, we present the method of moments, the nonequilibrium diffusion approximation, and the variable Eodington method. Finally, we discuss frequency dependence (Sec. VI), velocity-dependent terins ( Sec. VII), and the energy equation (Sec. VIII). Results using the code SPEC, which was derived from these considerations, are briefly discussed in Sec. IX.

II. THE EQUATIONS ON NONLINEAR PULSATION THEORY

The basic equations of nonlinear pulsation theory are

$$
\begin{aligned}
& \left(\frac{\partial r}{\partial t}\right)_{M_{r}}=u, \\
& \left(\frac{\partial u}{\partial t}\right)_{M_{r}}=-\frac{G M_{r}}{r^{2}}-4 \pi r^{2}\left(\frac{\partial P}{\partial M_{r}}\right)_{t}, \\
& \left(\frac{\partial F}{\partial t}\right)_{M_{r}}+P\left(\frac{\partial V}{\partial t}\right)_{M}=-\left(\frac{\partial L_{r}}{\partial M_{r}}\right)_{t}, \\
& V=\left(\frac{\partial}{\partial M_{r}}\right)_{t}\left(\frac{4 \pi}{3} r^{3}\right)
\end{aligned}
$$

and

$$
L_{r}=-\frac{\left(4 \pi r^{2}\right)^{2} u_{\sigma}}{3 k}\left(\frac{\partial T^{4}}{\partial M_{r}}\right)_{t} \text {. }
$$

where mass $\left(M_{r}\right)$, momentum $\left(M_{r} u\right)$, and energy $(E)$ are conserved. $L_{r}$ is the luminosity where $\sigma$ is the usual ac/4 ant $k$ the opacity. Early numerical schemes to solve these equations were developed iy Christ. ${ }^{4}$ and Cox, Brownlee, and Eilers. 5 The driving force for the pulsation is contained in the opacity and the equation of state, as described in the so-called gamma $(\gamma)$ and kappa $(x)$ mechanisms. A typical Rosseland opacity and EOS (equation of state) for the Cox-Davis I mixture* is shown in Figs. 1 and 2. The ionization level of hydrogen, for 


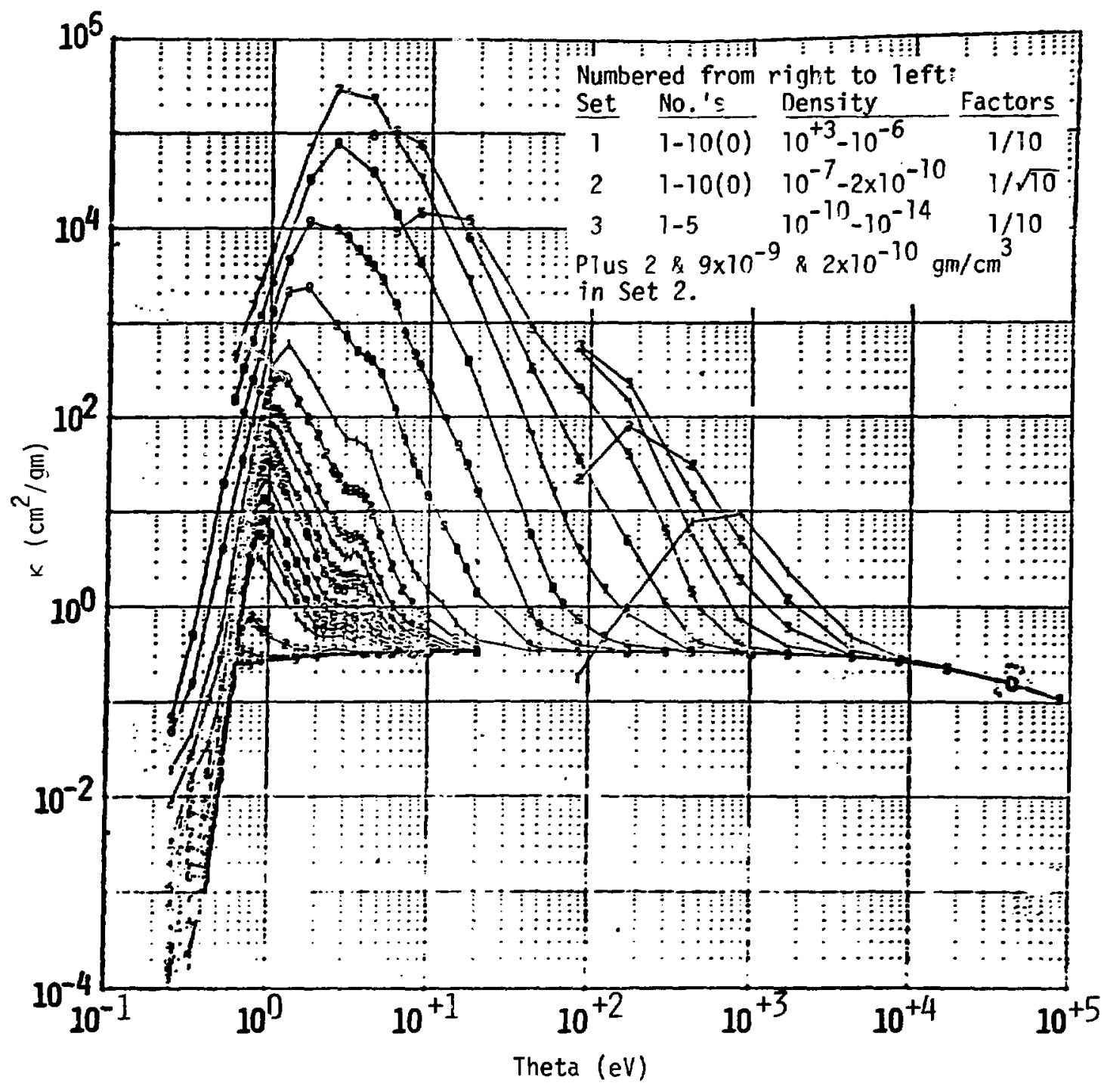

Fig. 1.

Rosseland opacity us temperature at selected densities for the Cox-Davis $I$ mixture $(X=0.602, Z=0.044)$. 


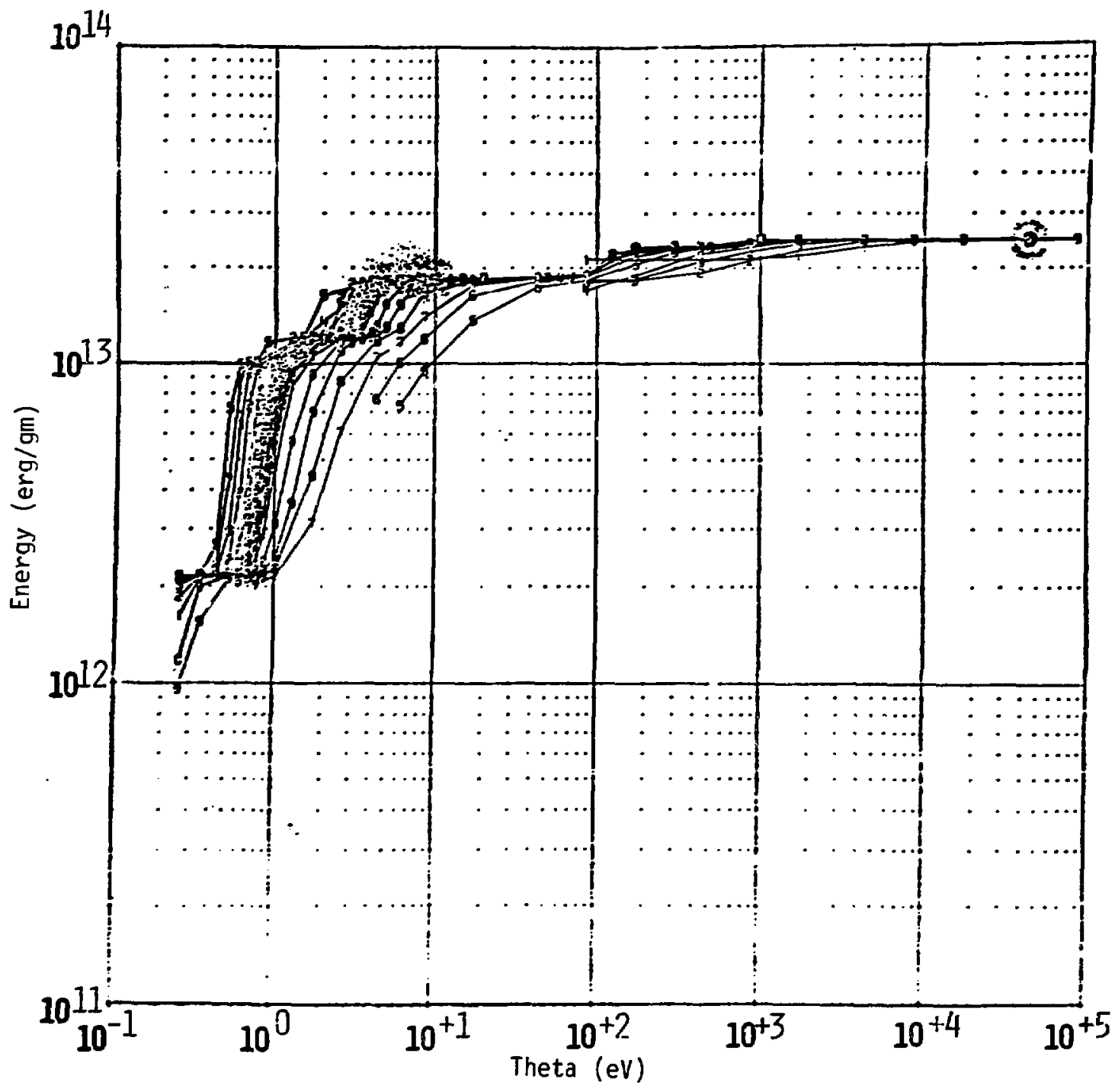

Eig. 2. Equation of state (energy) vs temperature at selected densities for the Cox-Davis I mixture. 
instance, is evidenced by a bump near $1 \mathrm{eV}$ (Fig. 1). In Christy's early work, these data were fit so that the ionization bumps were represented in the fits. From the earlier work of $\mathrm{Cox}$ et al. ${ }^{5}$ the tables were used for the opacity and equation of state of the astrophysical mixture. A considerable amount of linear pulsation theory has been developed using these tables or fits of the data in these tables. This work has resulted in the delineation of the blue edge of the instability strip giving the mode of pulsation and its growth rate (Q) as well as other insights into pulsation theory. Linear theory results will not be discussed here; however, the work is discussed in various papers by Bake. , 6 Iben, 7 and Cox and King. 8

The first attempts to apply multifrequency transfer theory to the methods of nonlinear pulsation were by Bendt and Davis, ${ }^{9}$ Castor, ${ }^{10}$ and Keller and Mutschlecner. 11 Prior to the se attempts, only diffusion theory had been used, even in the optically thin atmosphere that develops around the star. The variable Eddington approach was applied by Davis to nonlinear pulsation theory in 1969, before Auer and Mihalas ${ }^{12}$ applied it to static atmospheres. The application of the variable Eddington approach to many problems in astrophysics was one of the milestones in transfer theory during the 1970's (Auer, 13 Hsieh and Spiegel, ${ }^{14}$ Leung, ${ }^{15}$ Falk and Arnett, ${ }^{16}$ Schwartz, ${ }^{17}$ Harvel, ${ }^{18}$ Apruzese, ${ }^{19}$ and others).

It is apparent that some stars are pulsating in modes other than the fundamental mode and, in fact, in some cases the star is pulsating in more than one mode (double-mode Cepheids). It is also nossible for a star to pulsate in nonradial modes such as observed for the sun. Generally. Cepheids, RR Lyrae, and W Virginis stars are believed to pulsate only in the fundamental or first-overtone modes.

The overall description of the driving regions is shown in a plot of the work cycle for a typical pulsating star (Fig. 3). It appears that the blue edge of the instability strip in the usual Hertzsprung-Russell diagram depends on the saturation of this driving mechanism and possibly on dissipation of shock waves in the atmosphere. The limit at the red edge seems to depend on the presence of strong convection in the driving region of the star. We do not at present have a good theory for convection, particularly for time-dependent convection, so we do not have a good understanding for the theoretical location of the red edge. We avoid the issue of convection, assuming its effect on the transport of energy 


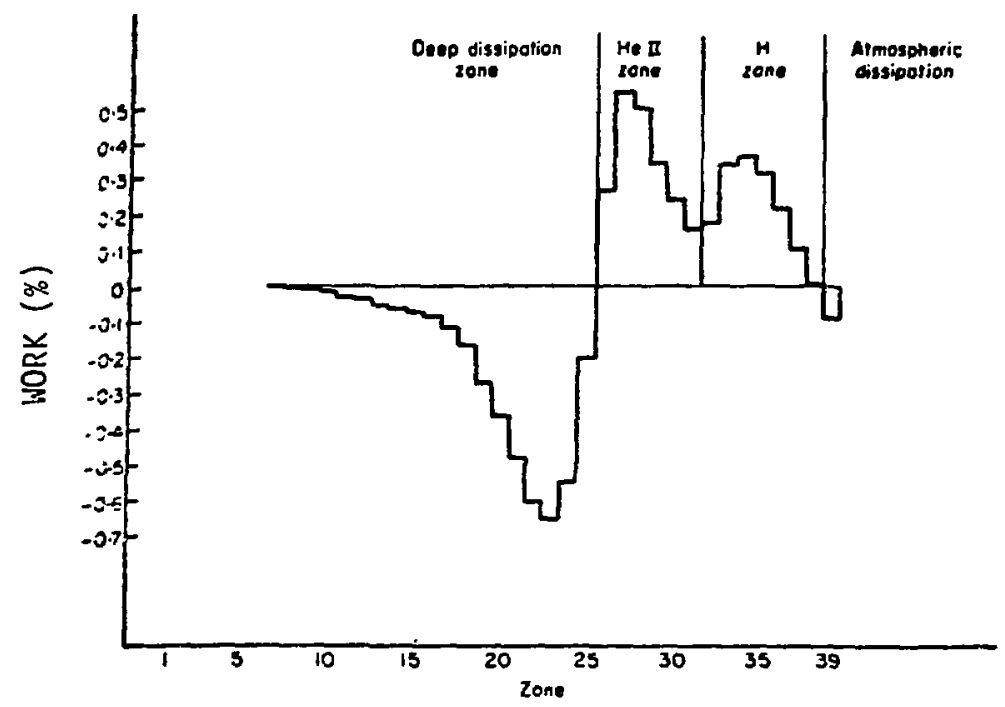

Fig. 3.

The work cycle vs zone number for a typical pulsating star.

in the outer regions of the star to be small. In the next section, we develop the diffusion equation from the spherical transfer equation (Sec. III).

III. THE RADIATION TRANSFER EQUATION

The diffusion equation used by Christy, ${ }^{4}$ Cox, Brownlee and Eilers, ${ }^{5}$ Baker and Kippenhan 20 and others may be derived from the fundamental transfer equation in spherical geometry,

$$
\begin{aligned}
\frac{1}{c} \frac{\partial I^{\nu}}{\partial t} & +\frac{\left(1-\mu^{2}\right)}{r} \frac{\partial I^{\nu}}{\partial \mu}+\mu \frac{\partial I^{\nu}}{\partial r}+\left(\sigma_{a}^{\nu}+\sigma_{S}^{\nu}\right) I^{\nu} \\
& =S^{\nu}+\sigma_{S}^{\nu} \int_{-1}^{1} P\left(\mu \cdot \mu^{\prime}\right) I^{\nu}\left(\mu^{\prime}\right) d_{\mu^{\prime}},
\end{aligned}
$$

where $I^{\nu}$ is the sprcific intensity along the ray with direction cosine $\mu$ and photon frequency $v$. The absorption and scattering coefficients are $\sigma_{a}^{\nu}$ and $\sigma_{s}^{\nu}$. the source function $S^{\nu}$, and the phase function for photon scattering $P\left(\mu \cdot \mu^{\prime}\right)$. The basic assumptions of diffusion theory are a slowly varying field (i.e.., $\partial I / \partial t=0 . n)$ and near isotropy, implying for the intensity 


$$
I=I_{0}+I_{1} r_{1}(\mu) \quad \text {; }
$$

therefore $E$, the radiation erergy density, is

$$
E=2 \pi \int_{-1}^{1} I d \mu=4 \pi I_{0},
$$

and $F$, the flux, is

$$
F=2 \pi c \int_{-1}^{1} I \mu d \mu=\frac{4 \pi}{3} c I_{1} .
$$

In addition, scattering is assumed isotropic [i.e., $\left.\underline{s}_{1}^{1} P\left(\mu, \mu^{\circ}\right) d_{\mu}^{\prime}=0.0\right]$. The results of integration of $\mathrm{Eq}$. (6) in terms of $E$ and $F$ over frequency, assuming the source function is Planckian $\left(S_{v}=E_{v}\right)$, is

$$
F=-\frac{4 \pi}{3} \nabla T \int_{0}^{\infty} d v \frac{1}{\sigma_{v}} \frac{\partial B_{v}}{\partial T},
$$

where $\sigma_{v}=\sigma_{a}^{\nu}+\sigma_{s}^{\nu}$. Tile final form for the equilibrium diffusion equation assumes radiative equilibrium (i.e.. $B=\int_{0}^{\infty} 4 \pi B_{v} d v=a T^{4}$ ), and is then

$$
F=-\frac{a c}{3 \sigma_{R}} \nabla T^{4}
$$

where

$$
\sigma_{R}=\frac{\int_{0}^{\infty} d v \frac{\partial B_{v}}{\partial T}}{\int_{0}^{\infty} d v \frac{1}{\sigma_{v}} \frac{\partial B_{v}}{\partial T}}
$$

and $\sigma_{R}$ is the Rossel and mean. 
IV. EXPANSION IN TERMS OF MOMENTS

In our calculation for the transfer of radiation, we use the method of moments. Again, an expansion of the intensity is made where the resulting moments, to second order, are

$$
\begin{aligned}
& M_{0}=2 \pi \int_{-1}^{1} I_{\mu} d_{\mu}=\frac{E}{c}, \\
& M_{1}=2 \pi \int_{-1}^{1} I_{\mu} \mu d_{\mu}=\bar{F},
\end{aligned}
$$

and

$$
M_{2}=2 \pi \int_{-1}^{1} I_{\mu}{ }_{\mu \mu} d_{\mu}=\frac{\overline{\bar{P}}}{c} .
$$

When these moments are introduced into Eq. (6), by integration, we get two equations,

$$
\nabla \cdot \bar{F}=-c_{\sigma_{a}^{-}}\left(E-\frac{4 \pi B(T)}{c}\right) \text {, }
$$

where $\sigma_{a}^{\prime}$ is the absorption coefficient corrected for induced emission, and

$$
c \nabla \cdot \overline{\mathrm{P}}=-\sigma_{\mathrm{T}} \bar{F} \text {. }
$$

where $\sigma_{T}=\left(\sigma_{a}^{\infty}+\sigma_{s}\right)$. The time-dependent terms and the frequency parameter $v$ have been dropped at present, for simplicity. The nonequilibrium diffusion transport approximation is obtained by closure of Eq. (10), with the assumption that

$$
\nabla \cdot \bar{P}=\frac{1}{3} \nabla E \quad ;
$$


i.e., the Eddington approximation (isotropic radiation energy field). The method allows for cooling of material by radiation in optically thin regions and diffusion through material with or without energy exchange with the material. Equations (9) and (10) are similar to those used by Auer and Mihalas ${ }^{12}$ where, in their notation,

$$
\frac{d H}{d \tau}+\frac{2 H}{k r}=-(J-S)
$$

and

$$
\frac{\partial K}{\partial \tau}+\frac{(3 K-J)}{K r}=-H
$$

Therefore, $J=c E, H=\bar{F}, K=c \overline{\bar{P}}$, and $d \tau=\rho k d r$. We will continue to use $E, \bar{F}$, and $\overline{\bar{p}}$, as defined in Eqs. (8). Auer ${ }^{13}$ has introduced a sphericity factor $q$, defined as

$$
\text { In } q=\int \frac{(3 f-1)}{f r} d r \text {. }
$$

where $f=K / J$ and then $E q$. (12) reduces to

$$
\frac{1}{q} \frac{\partial(f q J)}{\partial \tau}=-H
$$

We have not found it necessary to use this form of the flux equation.

In spherical coordinates our time- and frequency-dependent moment equations are

$$
\frac{\partial E_{v}}{\partial t}+\frac{1}{r^{2}} \frac{\partial\left(r^{2} F_{v}\right)}{\partial r}=c \sigma_{p}^{v}\left(\frac{4 \pi B_{v}}{a}-E_{v}\right)
$$

and

$$
\frac{1}{c} \frac{\partial F_{\nu}}{\partial t}+c\left[\frac{\partial f^{\nu} E_{\nu}}{\partial r}+\frac{\left(3 f^{\nu}-1\right)}{r} E_{\nu}\right]=-\sigma_{R}^{\nu} F_{\nu} \text {. }
$$


The Planck mean, $o_{p}$, is defined as

$$
\sigma_{p}=\int_{0}^{\infty} a_{\nu}^{-B}(T) d v / \int_{0}^{\infty} B_{v}(T) d \nu \text {, }
$$

and $f^{v}\left(=P_{V} / E_{V}\right)$ is the variable Eddington factor with 1 imits between $1 / 3$ and 1 . In general, we do not use the Planck mean $\left(\sigma_{p}\right)$ as it is usually 10 to 100 times the Rosseland mean and depends critically on the treatment of line widths. If $f=1 / 3$ and the radiation field is in equilibrium with the material energy field (i.e., $E_{r}=a T^{4}$ ), then $E q$. (14) again reduces to the well-known equilibrium diffusion equation [Eq. (7)] as expected. If the medium is plane parallel. for instance, then Eqs. (13) and (14) will reduce to

$$
\frac{\partial E}{\partial t}=-\frac{\partial F^{\prime}}{\partial x}-c \sigma_{p}(E-B)
$$

and

$$
\frac{\partial F}{\partial t}=-c^{2} \frac{\partial(f E)}{\partial x}-c o_{R} F
$$

Combining Eqs. (16) and (17) and assuming $o_{p}=\sigma_{R}=0.0$ (i.e., transparent), we obtain

$$
\frac{\partial^{2} E}{\partial t^{2}}=\bar{c}^{2} \frac{\partial^{2} E}{\partial x^{2}} \text {. }
$$

which is the wave equation, where $\bar{c}=c \sqrt{f}$. The correct 1 imit for optically thin media is achieved for $f=1$. These are the thick $(f=1 / 3)$ and thin $(f=1)$ limits of the variable Eddington approximation.

All that is required for a complete transfer solution using Eqs. (13) and (14) is the correct Eddington factor ( $f$ ). We find $f$ (next section) from a discrete ordinate solution of the plane geometry transfer equation in the static geometry or from a direct (Y-line) solution in spherical geometry. In the solutions of Auer and Mihalas, 12 is found by iteration. 


\section{v. THE VARIABLE EDDINGTON FACTOR}

As the radiation streams from the optically thick region into a vacuum, the Eddington factor will vary from a value of $1 / 3$ to 1 . To determine the Endington factor, therefore, we need a scheme that depends on optical depth and the location of the source. Imagine a spherical source (B) with radius a surrounded by a vacuum (Fig. 4). A simple solution for $f$ is shown in the following equations. Given

$$
\mu_{1}=\cos \theta_{1}=\sqrt{\left(1-a^{2} / r^{2}\right)} \text {. }
$$

and

$$
I(\mu)=\left\{\begin{array}{ll}
B & \mu>\mu_{1} \\
0 & \mu<\mu_{1}
\end{array} .\right.
$$

yielding

$$
\begin{aligned}
& E=\frac{2 \pi B}{c}\left(1-\mu_{1}\right), \\
& F=\pi B\left(1-\mu_{1}^{2}\right) .
\end{aligned}
$$

and

$$
P=\frac{2 \pi B}{3 c}\left(1-\mu_{1}^{3}\right) \quad ;
$$

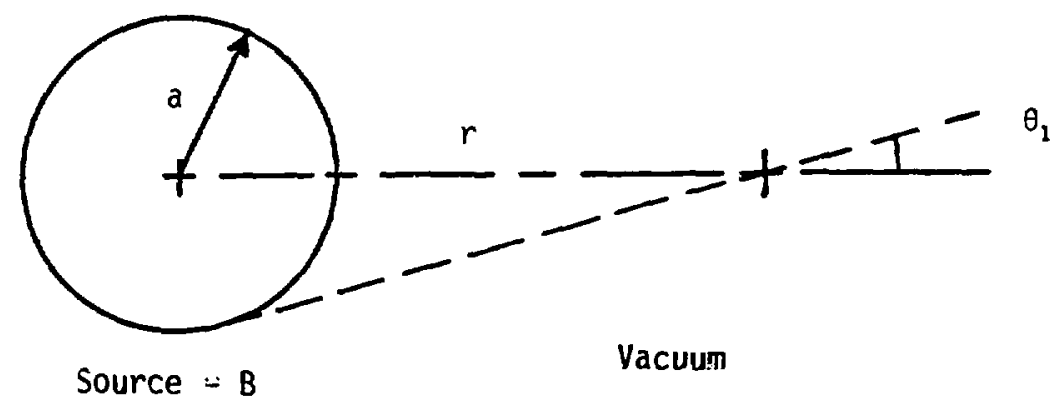

Fig. 4 .

The geometry for estimating a variable Eddington factor. 
then,

$$
i=\frac{P}{E}=\frac{1}{3}\left[1-\frac{2 F}{C E}+\left(\frac{2 F}{C E}\right)^{2}\right] \text {. }
$$

The actual method used in the evaluetion of $f$ in SPEC is the following. Assuming that the thin layer of atmosphere surrounding the star is planar, we apply a double Gaussian approximation to the solution of the transfer equation. The ray equation is

$$
\mu \frac{\partial I v}{\partial x}=\sigma_{v}^{-}\left(B v-I_{\nu}\right)
$$

or by integration.

$$
I_{i}=\tau_{i-1} \exp \left[-\left(\tau_{i}-\tau_{i-1}\right)\right]+\int_{\tau_{i-1}}^{\tau_{i}} B \exp \left[-\left(\tau_{i}-\tau\right)\right] d \tau \text {. }
$$

and by parts,

$$
\begin{aligned}
I_{i}^{\nu} & \left.\left.=\left(B^{\nu}-\frac{\partial B^{\nu}}{\partial \tau}\right)_{i}+\left[\frac{\partial B^{\nu}}{\partial \tau}\right)_{i}-\frac{\partial B^{\nu}}{\partial \tau}\right)_{i+1}\right] e^{-\Delta / 2} \\
& +\left[I_{i-1}^{\nu}-\left(B^{\nu}-\frac{\partial B^{\nu}}{\partial \tau}\right)_{i-1}\right] e^{-\Delta}
\end{aligned}
$$

where

$\tau=\sigma \Delta X, \Delta=\tau_{i}-\tau_{i-1}$,

$\Delta X$ is the slab thickness, and

$$
E^{v}=\sum_{1}^{K} A_{\mu} I_{\mu}^{\nu}, P^{v}=\sum_{1}^{K} A_{\mu}{ }^{2}{ }^{2} I_{\mu}^{v}
$$

where the $A_{\mu}$ 's are the Gaussian weights; 
then

$$
f^{\nu}=\frac{E^{\nu}}{p^{\nu}} .
$$

In an optically thick zone (i), Eq. (22) reduces in the limit to

$$
I \simeq B-\frac{\partial B}{\partial \tau} \quad \text {, }
$$

the diffusion approximation discussed in Sec. III. The se snapshot Eddington factors, which are frequency dependent, are formed every cycle in pilsation calculations. In other applications they are calculated every 10 or so cycles in order to reduce the computing time.

\section{FREOUENCY DEPENDENCE}

In nonlinear, multifrequency, radiation-transfer-coupled hydrodynamics calculations, it is necessary to consider the angular dependence of the radiation flow in conjunction with the rrequency dependence. Therefore, it is necessary to calculate the frequency-dependont Eddington factors along with the averaged monoch.omatic means. The frequency dependence of the flow is coupled with the hydrodymamic motion of the materials. Strong lines or ionization edges may occur in a region, causing emission and absorption effects to dominate the energy transfer. To resolve these effects, and still use only a reasonable number of frequency groups, we considered the relationship of these edges and the location of the peak of the Planck function in selecting frequency groups. By locating the band edge near the ionization jump and by adding frequency groups where the energy is being carried by the near-Planckian photon distribution, we $c$ an reduce the number of frequency bands necessary in a typical calculation. A particular selection of band edges that we use most frequently in our SPEC calculations is shown in the Appendix. The other frequency groupings are for snapshot spectra of colors from the SPEC structures. From the integration of our basic line data $[u(h v / k T)=0.05]$, we can then form the necessary Rosseland and Planck group means. As indicated, we seldom use the Planck means but have found a prescription for strong lines, called the thick/thin approximation, that works quite well. In this prescription, $k$ is replaced by $\sqrt{k_{r} k_{p}}$ and the source function $s$ by $s \cdot \sqrt{x_{p} / k_{R}}$ in the transfer equations. Other means 
(e.g., transmission means) havc been explored and result in some benefit for certain problems.

\section{VIT. VFLOCTTY TERMS AND RFTARDATION .}

As the velocity of motion increases, there is a greater shift from the frequency of the photons in the rest frame to that of the observers' frame of reference. There is also the effect of the finite speed of light, or the transit time of the photon, in the moving mesh. Both of these terms are small; but, in order to determine their effects on the solution of the transfer equations in pulsation theory, we did the following study. For the approximation of finite $c$ we must consider the added terms of $d E / d t$ and $(1 / c) d F / d t$. In our implementation of the nonequilibrium diffusion equation, it is possible to include these terms in the matrix elements for the implicit solution in a straightforward manner. A problem that does arise is from the $(1 / \mathrm{c}) \mathrm{dF} / \mathrm{dt}$ term, which can cause instabilities that affect the solution. We drop this term and include what is commonly called a "flux limiter" to retard flow. The flux limiter simply restricts the flow from a zone to be less than $c E$, the equilibrium limit. An understanding of this effect is shown in Fig. 5. The analytic solution shown is just a plane wave. oscillations that occur when $1 / \mathrm{c} \partial \mathrm{F} / \partial \mathrm{t}$ is included do not affect the location of the front, but the time step has been reduced by these excursions. As expected, the "retardation" effects were indisce.table in the light curves for normal pulsating stars.

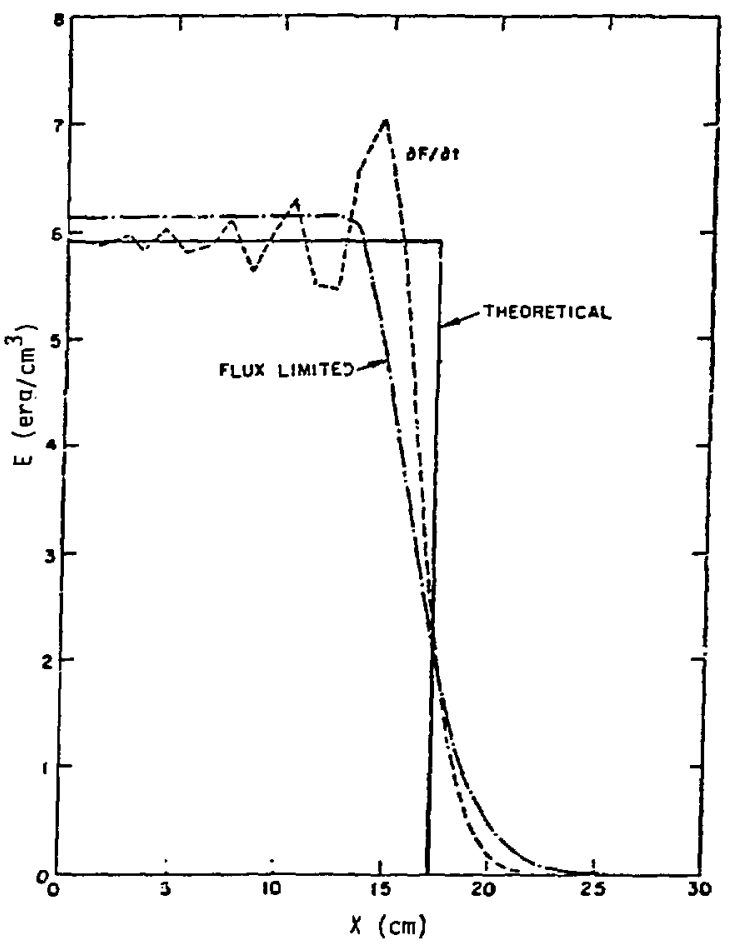

Fig. 5.

The radiation energy density for a wave transiting an optically thin slab usin? the $\partial F / \partial t$ term in the 2nd moment equation (--) and without $\partial F / \partial t$ using a flux limiter (-.-) compared to the analytic solution $(-)$. 
A proper derivation of the transfer equation, including relativistic effects to order $(u / c)^{2}$, has been carried out by Fraser. 21 We included these terms in a manner discussed by Freeman et al. and to the approximation proposed by Castor 22 for the treatment of stellar atmospheres (Davis). 23 The velocjty dependent, equations to order $u / c$ are:

Zeroth Moment

$\rho \frac{D\left(E_{v}^{0} / \rho\right)}{D t}+\nabla \cdot\left(\bar{F}_{\nu}^{0}+\bar{u} \cdot \bar{F}_{v}^{0}\right)=c \mu_{v}^{0}\left(\frac{4 \pi B_{v}^{\nu}}{\rho}-\bar{s}_{v}^{0}\right)-\mu_{\nu}^{0} \frac{\bar{u}}{c} \cdot \bar{F}_{\nu}^{0}$

First Moment

$$
\rho \frac{D\left(\bar{F}_{v}^{0} / \rho\right)}{D t}+c \nabla \cdot \overline{\bar{P}}_{v}^{0}=-\left(\mu_{v}^{0}+\mu_{s}\right) \bar{F}_{v}^{0}
$$

where $\overline{\mathrm{P}}_{v}^{0}=f E_{v}^{0}$ and the absorption coefficient $\left(u_{v}^{0}\right)$ and source function $\left(B_{v}^{0}\right)$ are assuned known in the comoving frame. The terns added for the transformation relationships to the observer's frame are

$$
\begin{aligned}
& E=E^{0}+\frac{2 u}{c^{2}} F^{0}, \\
& F=F^{0}+u E^{0}+u P^{\circ} .
\end{aligned}
$$

and

$$
P=P^{0}+\frac{1}{c^{2}}\left(u F^{0}+F^{0} u\right),
$$

Where the flux has been transformcd by adding $\bar{u} \cdot \overline{\bar{P}}$ and the other terms are dropped as $(u / c)^{2}$ dependent. These added terms have again bcen found to be unimportant for the class of pulsating stars we are studying. 


\section{THE ENERGY EQUATICN}

It is apparent that the calculation of the energy coupling with the material field, for frequency-dependent problems, is the most difficult aspect of nonlinear radiative transfer theory. In the optically thick region, inside the star, the diffusion approximation is the most appropriate limit. The correspondi.ug energy equation is

$$
\rho \frac{d E_{m}}{d t}=-\frac{1}{\rho} \nabla \cdot F_{R}+P \frac{d V}{d t} \text {, }
$$

where $\nabla \cdot F_{R}$ is the divergence of the flux. For optically thin regions one should use the approximation,

$$
\rho \frac{d E_{m}}{d t}=\int_{0}^{\infty} \sigma_{v}\left[B_{v}-I_{v}\right] d v+P \frac{d V}{d t} \text {, }
$$

W: 2 re the average of $\sigma_{\nu}$ over frequency gives the Planck mean. The problem is how to couple these terms in the transition region between optically thick and optically thin regions or groups. It is even more complicated if there are time shifts from the corresponding retardation terms. We assume that these terms are small (Sec. VIJ) and write

$$
0 \frac{d E_{m}}{d t}=\int_{0}^{\infty} \int_{0}^{4 \pi}\left(\alpha_{\nu} R_{\nu}+B \nu_{\nu}\right)_{\nu} d \omega d \nu+P \frac{d v}{d t} \text {, }
$$

where $\alpha_{v}$ could equal $1-\mathrm{e}^{-\tau \nu}$ and $\theta_{v}=1-\alpha_{\nu}$, for instance. The proper choice for $\alpha_{v}$ appears to be $1-\exp \left(-\tau_{v}^{2}\right)$, which correctly limits Eq. (30) to streaming and diffusion.

A radiation pressure term $\left(1 / c \rho \int_{0}^{\infty} d v u_{v}^{0} F_{v}\right)$ must al so be included in the momentum equation [Eq. (2)] and the resulting work term ( $\left.\bar{u} / c \int_{0}^{\infty} d v u_{v}^{0} \bar{F}{ }_{v}\right)$ in the energy equations [Eq. (28) or (29)]. The temperature update is usually 1$\lrcorner$ mited to two possible schemes, one called "partial temperature," the other "multifrequency/gray." Generally, we use the "partial temperature" approach. 


\section{A. The "Partial Temperature" Approach}

One assumes that there is not much coupling in frequency in the source function with optical depth. This approximation is accurate when scattering does not dominate, as assumed in our calculations, and the source temperature is not too high. The energy equation for each frequency group is

$$
\frac{D\left(b_{j} \phi\right)}{D t}+\frac{4 a \theta^{3}}{\rho C_{v}}\left\{b_{j}\left[\left(P_{m}+\frac{\partial E}{\partial \tau}\right) \rho \frac{d \tau}{d t}\right]+\left[c p_{j}\left(b_{j} \phi-E_{j}\right)\right]\right\},
$$

where is a partial temperature $\left(b_{j} \mathrm{~T}_{i}^{4}\right)$. The final energy change is then the sum of all the frequency energy changes.

\section{B. The "Multifrequency Gray" Approach}

In this approach we develop appropriate means from a multifrequency pass, which are then used in a gray formulation to update the temperature. The resultant equations are

$$
\begin{aligned}
& \frac{\partial E_{R}}{\partial t}+\frac{1}{r^{2}} \frac{\partial\left(r^{2} F_{R}\right)}{\partial r}=c \mu^{b} a \theta^{4}-c \mu^{\epsilon} E_{R} \text { and } \\
& \frac{1}{c} \frac{\partial F_{R}}{\partial t}+c\left[\frac{\partial\left(f^{0} E_{R}\right)}{\partial r}+\frac{\left(3 f^{0}-1\right)}{r} E_{R}\right]=-\mu^{0} F_{R},
\end{aligned}
$$

where

$$
\begin{aligned}
& \mu^{b}=\int_{0}^{\infty} \mu^{\nu} B_{v} d v / \int_{0}^{\infty} B_{v} d v, \\
& \mu^{e}=\int_{0}^{\infty} \mu^{\nu} E_{v} d v / \int_{0}^{\infty} E_{v} d v, \\
& f^{0}=\int_{0}^{\infty} f^{\nu} E_{v} d v / \int_{0}^{\infty} E_{v} d v,
\end{aligned}
$$


and

$$
\mu^{0}=\int_{0}^{\infty} \mu^{\nu} F_{\nu} d \nu / \int_{0}^{\infty} F_{\nu} d v
$$

are the gray average means. The multifrequency/gray approach does allow for a more natural limit to the diffusion equation in regions that are optically thick.

IX. THE "SPEC" CODE AND SOME APPLICATIONS

The methods discussed above for calculating the transfer of radiation in the atmosphere of a pulsating star have been incorporated into a code called "SPEC." In the limit of a nonequilibrium "gray" calculation, this code will give nearly the same results as the diffusion codes; for instance, FFF, the Cox, Brownlee, and Eilers code. ${ }^{5}$ The SPFC code uses the basic differencing-acrosszones scheme for the mean free path $\left(\lambda_{i}=1 / \sigma_{i}\right)$, as used by Christy ${ }^{4}$ and Cox, Brownlee, and Eilers ${ }^{5}$ :

$\sigma_{i}=\left(T_{i-1 / 2}^{4}+T_{i+1 / 2}^{4}\right) /\left(T_{i-1 / 2}^{4} / \sigma_{i-1 / 2}+T_{i+1 / 2}^{4} / \sigma_{i+1 / 2}\right)$

i.e., an inverse $T^{4}$ weighting .

The initial zoning for SPEC is obtained from a linearized solution of the pulsation equations and the static atmosphere in a code called LNA The zoning is developed in a geometric progression with a ratio like 1.2 but, in our case, we use more thin zones on the outside than are normally used in the diffusion codes. The boundary conditicns are either $P=0.0$ or the Castor imaginary corona condition, where $P$ is a function of radius. The pseudo viscosity is also cut off in the center of the mesh to reduce artificial dissipation. For stability, we have found it advantageous, as has Christy, to include some viscosity in the center zone during the calculations. This small addition of viscosity in the middle does not seem to affect the results but stabilizes the inner zone in the calculation.

Using the mesh developed from the linear code, we apply a fundamental mode in velocity space to initialize the pulsation. The value we normally use is from Christy's work and is 


$$
u_{i}=u_{0}\left(R_{i} / R_{0}\right)^{6}
$$

The above velocity field is applied at the times of expansion and removed when the star reaches near-limiting amplitude. The impuise is removed to allow the star to reach freely its limiting amplitude. The process takes approximately 25 minutes on the $\operatorname{CD} 77600$ computer. After reaching limiting amplitude, in the gray diffusion approximation, the multifrequency variable Eddington calculation is carried out until the star again attains limiting amplitude (approximately 25 more minutes of 7600 time).

The code (SPF.C), as applied originally to Cepheids, ${ }^{24}$ has also been applied to $W$ Virginis ${ }^{25}$ and to a model of an RR Lyrae star (SW And). 26 The details of these calculations are included in a number of papers published since 1965 and will not be discussed here except in the conclusions. For cepheids, the application of a detailed transfer theory seems to make little difference in the development of the light or velocity curves. This result is supported by the subsequent work of Keller and Mutschlecner. ${ }^{11}$ However, for $W$ Virginis, the effects are more dramatic. We find that by the inclusion of multifrequency transfer effects we can obtain the standstill on the declining part of the light curve, which does not appear in diffusion theory but is observed (Fig. 6). A strong shock forms in the atmosphere of $\mathrm{W}$ Virginis as observed in line doubling, the $\mathrm{H}$ and $\mathrm{K}$ emission lines of $\mathrm{CA} I I$, and hydrogen emission. For RR Lyrae, we believe we can show that the uv excess, as observed for SW And, is due to the inclusion of radiative transfer effects (Fig. 7).

\section{CONCLUSTONS}

Improved radiative transfer methods in nonlinear pulsation theory appear to be important in modeling RR Lyrae and $W$ Virginis stars and not so impartant in models of classical Cepheids. The usual equilibrium diffusion approximation works well in models constructed of classical Cepheids. The variable Eddington method is computationally fast and, with the appropriate Eddington factors, a complete transfer approximation can be obtained. 


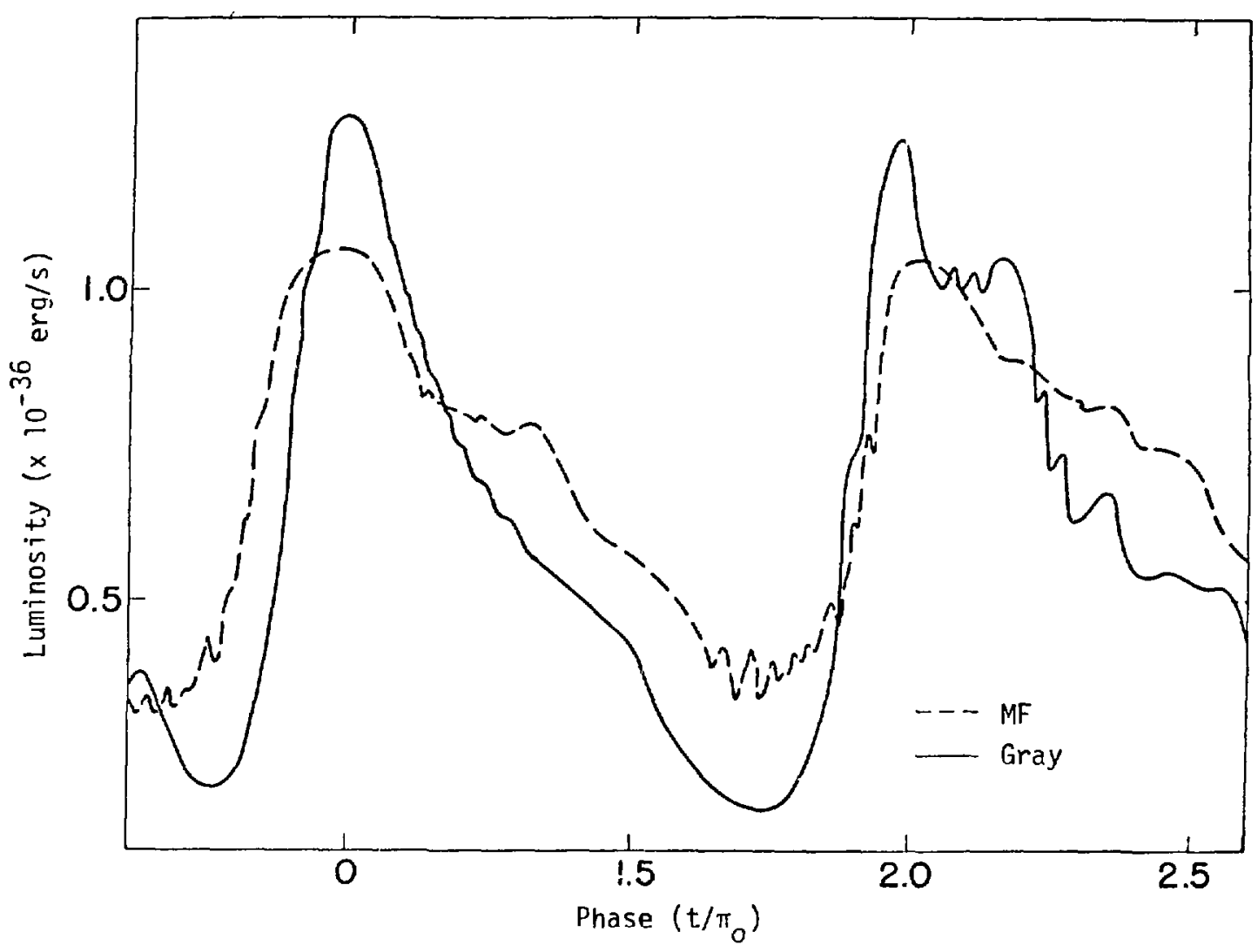

Fig. 6 .

The calculated Iuminosity vs phase for $W$ Virginis.

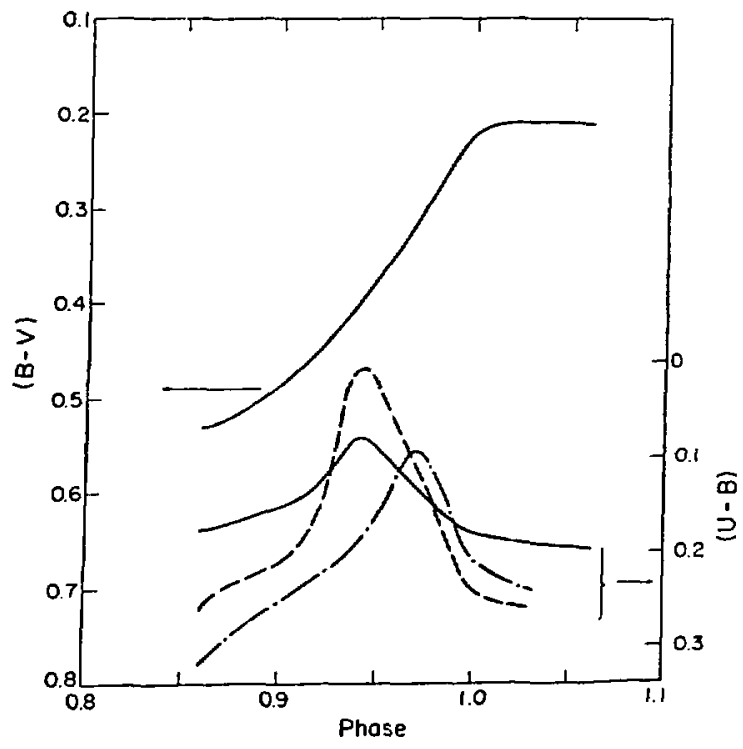

Fig. 7 .

The calculation of colors and observations for SW And during the phase in which a shosk is transiting the atmosphere. The $(U-B)$ colors from the gray (- $)$ and multifrequency (-) transfer stmuctures are compared to the observar'.ens (-). 


\section{REFERENCES}

1. A. S. Eddington, The Internal Constitution of the Stars, (Cambridge University Press, Cambridge, 1926).

2. S. A. Zhevakin, "Pulsating Stars," Russ. Astron. in. 30, 161 (1953).

3. J. P. Cox and C. A. Whitney, "Stellar Pulsation IV. A Semiclassical Period-Luminosity Relation for Classicai Ceptelds," Astrophys. J. 127, 561 (1958).

4. R. F. Christy, "A Model of W. Virginis with RV Tauri Characteristics," Astrophys. J. 145, 337 (1966).

5. A. N. $\operatorname{Cox}$, R. R. Brownlfe, and D. D. Eilers, "Time-Dependent Method for Computation of Radiation Diffusion and Hydrodynamics." Astrophys. J. 144. No. $3(1966)$.

6. N. Baker, Stellar Evolution, R. W. Stein and A. G. W. Cameron, eds. (Plenum Press, New York, 1967).

7. I. Iben, Jr., "On the Specification of the Blue Edge of the RR Lyrae Instability Strip," Astrophys. J. 166, 131 (1971).

8. J. P. Cox and D. S. King, "Evolution of Pop. II Stars," (Dudley Obs. Report No. 4) (1972).

9. J. E. Bendt and C. G. Davis, "A Radiative Transfer Model of a Cepheid," Astrophys. J. 169, 333 (1971).

10. J. I. Castor, "Atmospheric Dynamics in a Model RR Lyrae Star," Ph.D. Dissertation, California Institute of Technology (1966).

11. C. F. Keller and J. P. Mutschlecner, "Atmospheres of Classical Cepheid Variables," Astrophys. J. 161, 217 (1970).

12. L. H. Auer and D. Mihalas, "On the Use of Variable Eddington Factors on Non-LTE Stellar Atmospheres Computations," Mon. Not. R. Astron. Soc. 149, 65 (1970).

13. L. H. Auer, "The Stellar Atmospheres Problon," J. Quant. Spectrosc. Radiat. Transfer 11. 572 (1971).

14. S. H. Hsieh and E. A. Spiegel, "The Equations of Photodynamics," Astrophys. J. 207, $244(1976)$.

15. C. M. Leung, "Numerical Solution of the Radiative Transfer Equation in Spherically Symmetric Dust Shells," J. Quant. Spectrosc. Radiat. Transfer $16,559(1976)$.

16. S. W. Falk and W. D. Arnett, "Radiation Dynamics Envelope Ejection and Supernova Light Curves," Astrophys. J. Supp. 33. 515 (1977). 
17. R. D. Schwartz, "Radiative Transfer in Gray Circumstellar Dust Envelopes, vY Canis Majoris Revisited," Astrophys. J. 196, 745 (1975).

18. C. A. Harvel, "Radiative Transfer in Circumstellar Dust Shells," Astrophys. J. $210,852(1976)$.

19. J. P. Apruzese, "Radiative Transfer in Spherical Circumstellar Dust Envelopes. V. Theoretical Circumstellar Graphite and Silicate Enission Spectra," Astrophys. J. 207. 709 (1976).

20. N. Baker and R. Kippenhahn, "The Pulsations of Models of Delta Cepheid Stars. IJ." Ast.rophys. .' 142, 868 (1965).

21. A. R. Fraser, "The Fundamental Equations of Radiation Hydrodynamics," Atomic Weapons Research Establishment report AWRE 0-82/65, Aldermaston, England (1965).

22. J. I. Castor, "Radiative Transfer in Spherically Symmetric Flows," Astrophys. J. 178, 779 (1972).

23. C. G. Davis, Relativistic Terms in Nonlinear Pulsation Theory," Astrophys. J. 187, 175 (1974).

24. C. G. Davis, "Theoretical Models of 10-Day Cepheids," Astrophys. J. 221, 929 (1978).

25. C. G. Davis, "A W Virginis Model Using Radiation Transfer," Astrophys. J. 172, $419(1972)$.

26. C. G. Davis, "Non-Linear Radiative Transfer Model of SW Andromadae," The Proceedings of IAU Colloquium "29, W. S. Fitch, ed. (Pub, ishing House of the Hungarian Academy of Sciences, Budapest, Hungary, 1976). 


\section{APPENDIX}

TABLES USED IN ASTROPHYSICAL CALCULATIONS

FREQUENCY LROUP BOUNDARTES ( $\mathrm{eV}$ )

\begin{tabular}{|c|c|c|c|}
\hline & SPEC & Sne & \\
\hline & & Coarse & Fine \\
\hline No. & 13 & 20 & 20 \\
\hline 1 & 0.25 & 0.25 & $\overline{1.85}$ \\
\hline 2 & 0.50 & 0.50 & 1.91 \\
\hline 3 & 1.00 & 0.75 & 1.97 \\
\hline 4 & 1.50 & 1.00 & 2.03 \\
\hline 5 & 2.00 & 1.25 & 2.10 \\
\hline 6 & 2.50 & 1.50 & 2.17 \\
\hline 7 & 3.00 & 1.75 & 2.25 \\
\hline 8 & 3.40 & 2.00 & 2.34 \\
\hline 9 & 4.90 & 2.25 & 2.43 \\
\hline 10 & 5.90 & 2.50 & 2.53 \\
\hline 11 & 8.50 & 2.75 & 2.64 \\
\hline 12 & 13.68 & 3.00 & 2.75 \\
\hline 13 & 30.00 & 3.20 & 2.88 \\
\hline 14 & & 3.40 & 3.02 \\
\hline 15 & & 3.75 & 3.18 \\
\hline 16 & & 4.10 & 3.35 \\
\hline 17 & & 4.90 & 3.54 \\
\hline 18 & & 5.90 & 3.76 \\
\hline 19 & & 8.50 & 4.12 \\
\hline 20 & & 13.68 & 4.28 \\
\hline
\end{tabular}

